\title{
LA DIMENSIÓN AFECTIVA DEL APRENDIZAJE DE LAS CIENCIAS Y LAS RELACIONES CTS
}

\author{
Lic. Lucas Guillermo González de la Barrera \\ Lic. Ana Cecilia Mazarío Triana \\ Lic. Israel Mazarío Triana \\ Universidad Camilo Cienfuegos, Matanzas. Cuba
}

\begin{abstract}
The aim of the present work is to comment how on the most recent developments in the field of relations Science-Technology society have led to significant chances in daily classroom practice. Researchers and tea chers jo scientific education have claimed the necessity to offer courses, lectures and ro rams about social aspects the sc/ence and technology with the goals to obtain a benefit, having as a starting point the activity of the proffesional future. A synthesis of the concepts and theories that support the framework of this une of the concepts and theories that support the framework of this une of investigation is given.
\end{abstract}

\section{RESUMEN}

Con el presente trabajo se pretende divulgar como el desarrollo en el campo de las relaciones CTS han producido cambios significativos en la práctica de la tarea docente. Investigadores y docentes en educación científica expresan la necesidad (preocupación) de ofrecer conferencias, cursos y programas sobre los aspectos sociales de la Ciencia y la tecnología con el objetivo de obtener beneficios al tomar síntesis de conceptos y teorías que fundamentan la estructura de esta línea de investigación es dada.

\section{INTRODUCCION}

El mundo actual se encuentra sumido en una gran revolución sociocultural basada en la ciencia y la tecnología. Es por ello que la forma de pensar y actuar de los científicos haya pasado a formar parte de las distintas ramas de la producción, de los servicios y de la sociedad. Por ello, el mundo laboral de hoy requiere de personas con conocimientos, instrumentaciones, actitudes y valores acorde con estas exigencias.

En los últimos años han tenido un enorme desarrollo los estudios de las relaciones ciencia, tecnología y sociedad (CTS), sobre todo desde la educación científica, lo cual queda evidenciado por la gran cantidad de trabajos, artículos y de revisiones bibliográficas publicadas, de ahí que los trabajos sobre estos temas constituyan en la actualidad una línea de investigación importante en didáctica de las ciencias.

Es por ello que las universidades, así como los diferentes sistemas educacionales, se preocupan por ofrecer cursos, asignaturas y programas sobre los aspectos sociales de la ciencia y la tecnología, pues con ello se pretende formar a los estudiantes para que sepan desenvolverse en un mundo inundado por los desarrollos científicos y tecnológicos, para 
que sean capaces de adoptar actitudes responsables y tomar decisiones fundamentales frente a esos desarrollos y sus consecuencias.

De ahí que a la consecución de este objetivo de alfabetización científica de todos los individuos se le esté concediendo cada vez mayor importancia.

Diversas investigaciones han mostrado que en la enseñanza de las ciencias se confrontan numerosos problemas, en consecuencia a lo anterior se hace necesario implicar a los docentes en los procesos de cambio, si se pretende que esto se generalice, para que de esta manera el profesorado se apropie de las nuevas orientaciones y comprenda la importancia de los nuevos contenidos, de los nuevos objetivos y finalidades de la educación científica, imprescindibles para formar ciudadanos capaces de vivir al nivel de su tiempo.

\section{DESARROLLO}

Para todo docente y en especial para los que se dedican a la enseñanza de las ciencias, es muy importante tener presente la siguiente frase que data de fines del siglo XIX:

Educar es depositar en una persona toda la obra humana que le ha antecedido, es hacerle resumen del mundo viviente, hasta el día en que vive, es ponerla al nivel de su tiempo para que flote sobre él y no debajo de su tiempo, con lo que no podría salir a flote, es prepararla para la vida.

(José Martí) 1968

Los conocimientos científicos que hoy forman parte de la cultura humana, y que son aprendidos por los estudiantes en los centros educacionales, no pueden llegarle desde afuera y ser aprendidos como cadenas verbales, o sea, como una unión consecutiva, estricta e inalterable de palabras o frases, por la general afuncional, como sucede hoy en la mayoría de los casos.

Los fundamentos sobre los que descansan el aprendizaje de los conocimientos científicos y su enseñanza, así como las principales características y los procedimientos fundamentales para la obtención de este tipo de conocimiento, aún no son del dominio de la mayor parte de los profesores. Parlo que no puedan lograr, con su enseñanza, el aprendizaje de estos conocimientos por parte de los estudiantes, sino solo trasmitirlos de manera ya acabada, y hacer que los fijen como cadenas verbales a partir de su aplicación mecánica. Es por ello que el conocimiento científico debe ser construido por el estudiante, cuando establece las relaciones más generales de ese conocimiento. En otras palabras, estos conocimientos deben ser aprendidos par el estudiante a partir de su actuación.

Toda la vida de una persona transcurre a través de su actuación, por actuación vamos a entender la interacción que la persona establece a través de su actividad y comunicación con los objetos y sujetos de un determinado contexto y por contexto, entendemos un determinado medio natural y / o social en una época histórica específica.

Hasta hace muy poco tiempo, las preocupaciones curriculares se centraban casi exclusivamente en la adquisición de conocimientos científicos, con el fin de familiarizar a 
los estudiantes con las teorías, conceptos y procesos científicos, en la actualidad estas tendencias están cambiando, al introducir en los currículos aspectos que orientan socialmente la enseñanza de las ciencias y tratan de relacionarlas con el propia estudiante (Hodson, 1993, Bybee y Deboer,1994 ,Bybee y Ben-Zui, 1998).

Como hemos señalado anteriormente, la alfabetización científica y tecnológica es una de las finalidades planteadas en muchas de las reformas curriculares que se están llevando a cabo en numerosos países, pera tal y coma la demuestran algunos trabajos en este sentido, gran parte del profesorado, que debe llevar a vías de hecha estas reformas, no comparten algunos de sus objetivos y finalidades (Vilches et al., 1999). Muchos orientan su enseñanza hacia la preparación de cursos superiores, es decir, hacia la formación de futuros científicas, no teniendo en cuenta que de lo que se trata es formar básicamente a todas las personas (científicos y no científicas), de modo que la gran mayoría de la población pueda disponer de los conocimientos y destrezas necesarias para desenvolver-se en la vida diaria, ayudar a resolver problemas y necesidades de salud personal y supervivencia global, adaptar actitudes responsables frente al desarrolla y sus consecuencias, así cama poder participar activamente en la toma de decisiones. Esta discusión es un paso previo necesario para la comprensión del papel fundamental de las interacciones ciencia, tecnología y sociedad en la consecución de los objetivos y finalidades de la educación científica.

Con el objetivo de lograr las finalidades antes señaladas, se requerirá que la ciencia que está presente en el currículo incluya objetivos y contenidos conceptuales, es decir, conocimientos científico -técnicos necesarios para que las personas puedan desenvolverse en un mundo cada vez más impregnada por el desarrollo científico y tecnológico actual. Es necesaria también incluir objetivos y contenidos procedimentales, que permitan aprender la que es la ciencia y la tecnología y cómo trabajan, para razonar y resolver mejor los problemas de la vida cotidiana. Pero también hay una dimensión afectiva en los objetivas a lograr en la enseñanza aprendizaje de las ciencias.

Esta dimensión afectiva se concreta en objetivas actitudinales y se relaciona con la finalidad de conseguir despertar el interés y el gusto por los estudias científicos en los alumnos. De ahí que el currículo ha de conformar creencias, actitudes y valores que, fundamentalmente, desarrollen un interés crítico por la actividad científica, actitudes y valores que permitan evaluar en el futuro el papel que la ciencia desempeñé y ha desempeñado en nuestras vidas y de esa manera preparar el camino para la participación colectiva en la solución de los problemas con los que se enfrenta la sociedad.

En las recientes reformas curriculares que se están desarrollando en las ciencias, la importancia social del tema de las actitudes es bien reconocida, pues en todas ellas los diseñadores del currículo han incluido explícitamente objetivos y contenidos actitudinales, por la que se hace necesario que las mimas se lleven al aula por parte del profesorado, que son en definitiva los que ponen a punto dichos currículos.

Es frecuente oír que los estudiantes llegan desmotivados y sin interés a las clases de ciencia, sin embargo, es difícil encontrar en nuestros programas, referencias directas o indirectas a objetivos o actividades de tipo actitudinal que ayuden a paliar este problema (Furió y Vilches, 1997). Es decir el profesorado reconoce la importancia vital de la motivación y de las actitudes, coma motores impulsores del aprendizaje de las ciencias pero, una vez en el aula, se olvidan de ellas. 
A pesar de que la investigación en el dominio afectivo del aprendizaje de las ciencias es relativamente nueva, últimamente está teniendo una creciente importancia, tal interés radica en que va a permitir plantear toda una serie de cuestiones que van a condicionar el trabajo en el aula, lo cual conllevará a una mejor comprensión de la necesidad de introducir las actividades de ciencia, técnica y sociedad en las clases de ciencia.

Muchas investigaciones en este sentido han encontrado que, en la educación obligatoria, a pesar de que se imparten cursos de ciencia con sistematicidad, estos no logran incentivar, como sería razonable pensar, el interés de los estudiantes por estas disciplinas.

Lo anterior resulta una gran problemática sobre la que debemos seguir insistiendo, pues desde hace casi un siglo, el profesorado ha considerado conveniente la implementación dentro del currículo de las ciencias $\mathrm{y}$, después de tanto tiempo, la enseñanza de estas disciplinas científicas, todavía las mismas no son capaces de incentivar en los estudiantes hacia su estudio, pues como se conoce de investigaciones que se han realizado en este sentido, en los niños y niñas de la enseñanza primaria, si existe, de manera muy generalizada, un interés y una curiosidad inicial por el mundo científica, el cual va decreciendo y manteniéndose relativamente bajo durante la etapa de enseñanza secundaria y preuniversitaria.

Es bueno señalar que en estos momentos muchos investigadores tratan de encontrar respuesta a esa problemática, lo cual queda evidenciado por la gran cantidad de trabajos que se han realizada y se realizan en este sentido (Schibecci, 1984,1986, Penick y Yager, 1986, Aikenhead, 1987, Boyer y Tiberghien, 1989, Ryan, 1990, Simpson y Q!iver, 1985, Vázquez y Manassero, 1995, 1997).

La discusión de esta cuestión es importante, pues el profesorado enfrascado en la mima puede hacer referencia a toda una serie de posibles causas de la actitud de desinterés de los estudiantes hacia las ciencias, y permitirá comprender la necesidad de introducir las interacciones CTS en su enseñanza.

Una gran parte de los profesores son del criterio de que estas actitudes negativas de los estudiantes hacia las ciencias son consecuencia de factores externos al proceso de enseñanza-aprendizaje y mencionando dentro de las mismas las siguientes:

$>$ Procedencia social de los alumnos.

$>$ Mayor interés hacia los medios masivas de comunicación.

$>$ Disfunciones existentes entre la capacidad intelectual de los estudiantes y el aumenta de las dificultades de los estudios científicos, particularmente de la física a medida que se eleva el nivel del curso(Gil et al, 1991)

Como se puede apreciar, en ningún momento son tenidos en cuenta factores internas del proceso de enseñanza aprendizaje que pueden también influir negativamente en la dimensión afectiva hacia el aprendizaje de las ciencias como son:

$>$ El clima del aula y del centro.

$>$ El tipa de enseñanza.

$>$ La actitud y expectativas del profesorado hacia el éxito de los alumnas.

Lo novedoso de las investigaciones didácticas que hemos citado plantean que la escuela es, al menos parcialmente responsable de la formación de actitudes pasivas 
hacia el aprendizaje de las ciencias, y señalan como características de la enseñanza que pueden contribuir a ello las siguientes:

$>$ Las finalidades de la enseñanza se reducen a que las estudiantes aprendan sola conocimientos científicos, sin tener en cuenta su desarrolla afectivo.

$>$ Escasa preocupación del profesorado por incidir de forma explícita en el interés de la ciencia coma vehículo cultural.

$>$ Presentación de estas materias como algo abstracto y excesivamente formal.

$>$ Imagen deformada que se presenta habitualmente de los científicos y de la ciencia, sin conexión can los problemas reales del mundo que nos rodea, es decir, sin tener en cuenta aspectos históricos, sociales (Bernal, 1967), ecológicos, etc. Ahí reside en cierta medida, el origen de mucho de los estudios de CTS en educación y en el campo de la filosofía de la ciencia.

Cada día que transcurre está lleno de nuevos descubrimientos en la ciencia. Los avances en el campo de la física, la matemática, la biología y la química son innegables y la cantidad de nuevos conocimientos por intervalo de tiempo se hace cada vez mayor.

Actualmente, es imposible poder dar todos los conocimientos que tiene la humanidad en cualquiera de estas ciencias en el corto espacio de uno o varios cursos escolares El impacto que la ciencia ha tenido y tiene en la vida de los hombres y mujeres, hace pensar en el interés por su estudio, en su enorme potencial didáctico, sin embargo, a pesar de la inclusión cada vez mayor del estudio de la ciencia en los niveles básico y medio, la actitud hacia ella no es la esperada.

Esto no es una tarea sencilla, se requiere de activar el pensamiento del estudiante, de hacerlo copartícipe de nuestros intereses por la ciencia, del deseo de saber, de querer investigar lo desconocido para él, no sólo en la clase sino fuera de ella en su tiempo libre. Unido a esto la creciente influencia de la escuela en la comunidad hace que los métodos de enseñanza desempeñen un papel decisivo en el aprendizaje de las ciencias.

Todas las causas citadas han dado lugar a estudias en los diferentes campas de la investigación en didáctica de las ciencias, que confluyen en el intento de cambiar y mejorar la actitud de los alumnos hacia la ciencia y su enseñanza.

La veracidad de las conocimientos científicos siempre debe ser demostrada, no solamente con el experimento sirio también en la práctica social, a sea, al ser aplicada a otras ciencias, la técnica y la sociedad en general, debido a que la práctica social es la que más repercute en la sociedad y par ende en los estudiantes.

En la práctica de la enseñanza de las ciencias, los profesores hacen más hincapié en mostrar la veracidad de un experimento, y en la mayaría de los casos, no plantean su introducción en la práctica social, siendo esta precisamente lo que más interesa a los estudiantes y una de las causas de que vean la ciencia divorciada de su mundo, convirtiéndose lo anterior en una de las posibles razones del desinterés hacia las ciencias, su estudio y de las actitudes negativas de los estudiantes hacia la misma.

En investigaciones llevadas a cabo con profesores y estudiantes de ciencias, los mismos coinciden en señalar la importancia de dichas interacciones para lograr un mayor interés en el alumnado hacia el estudio de las ciencias (Solbes y Vilches. 1992, 1995). 
Análisis realizadas en cursas de ciencia considerados de excelencia, realizados por estudiantes norteamericanos, Penick y Yager (1986) señalaron que las relaciones CTS constituían una parte central de los cursas más valorados, de igual forma comprobaron que los cursas con estas características, además de favorecer el interés, mejoraron las resultadas de las exámenes oficiales. Parlo que con lo anterior puede confirmarse el impartan-te papel que el tratamiento de las relaciones CTS puede desempeñar en el aumenta del interés de los estudiantes hacia la ciencia y su estudio.

El campo de las investigaciones de las interacciones CTS ha confluido en los últimos años con los señalamientos anteriores, es decir, con el estudio de las actitudes de los estudiantes hacia la ciencia y su aprendizaje, y también con las nuevas tendencias curriculares que promovían la alfabetización científica o ciencia para todos. En los dos casos, el tratamiento de las interacciones CTS puede ser un buen instrumento para la consecución de las finalidades de estas propuestas didácticas.

El objetivo básico del denominado movimiento CTS, es resaltar la necesidad de relacionar la ciencia y la tecnología can el medio natural y social. De ahí la necesidad de recuperar los aspectos socio-históricos, de las relaciones CTS, que permiten una visión contextualizada de la ciencia y suministran una elevada motivación hacia su estudio.

Las interacciones CTS se plantean cada vez más como una relación necesaria entre el aprendizaje de las ciencias y el medio exterior, es decir, coma una profundización del conocimiento científico, en los problemas asociados a su construcción, ya que el trabajo científica como cualquier otra, actividad humana, no tiene lugar aisladamente sino en un determinado medio social que afecta necesariamente dicho trabajo. De la misma manera, las circunstancias históricas del momento en que se desarrollan influyen en él, por ello el conocer la relación del conocimiento científico con el exterior, los problemas que el desarrollo científico y tecnológico genera a resuelve, va a permitir a los estudiantes y, en su caso, a los futuros científicos, tener una visión de la ciencia más completa y más contextualizada socialmente (Vilches y Furió, 1999).

Las transformaciones ocurridas en nuestras sociedades, los nuevos riesgos a escala global de algunos desarrollos, el papel de la ciencia y la tecnología como elementos estratégicas, motivan aún más los estudios en ciencia y tecnología y su evaluación.

Comprender las complejas relaciones CTS se convierte en algo necesario, si se quiere que en el futuro las personas tengan que tomar decisiones, adoptar actitudes responsables frente al desarrollo y las consecuencias derivadas de él.

Resulta pues extremadamente peligroso el hecha de que muchos de nuestros educandos y ciudadanos en general ignoren lo que significa la contaminación atmosférica, el calentamiento global, el daño a la capa de ozono, la desaparición de especies, los problemas del uso indiscriminado de algunas fuentes de energía, el mal manejo de desechos industriales, el incremento de los residuales que generan las grandes urbes, del hambre, de las condiciones de vida de los más pobres. Si desconocen todas estos y muchos otras problemas así como su impacto en el futuro, ¿cómo podrán tomar decisiones e incidir en las políticas de sus respectivos países? Es por ella que en la actualidad el analfabetismo científico y tecnológico es mucho más peligroso que en cualquier situación anterior.

Por toda lo anteriormente expuesta, que se hace necesario la inclusión de los programas de CTS, en los currículos de los diferentes niveles de enseñanza, para 
contribuir de esta manera no sólo a mejorar la actitud y el interés hacia la ciencia y su aprendizaje, sino también va a permitir aprender más ciencia y saber más sobre ella, al mostrar una imagen más completa y contextualizada de esta disciplina.

\section{BIBLIOGRAFÍA}

Aikenhead, G. S. (1985): Collective decision making in the social context of science. Science Education, 69: 453-475.

Aikenhead, G.S. (1987): High-School graduates beliefs about Science-TechnologySociety. III. Characteristics and limitations of scientific knowledge. Science Education, 71(4): 459-497.

Aikenhead, OS. (1988):An analysis of Four Ways of Assessing Student Beliefs about STS Topics. Journal of Research in Science Teaching, 25 (8): 607-624.

Bernal, J. D. (1967): Historia Social de la Ciencia. Península, Barcelona, vols. 1 y 2.

Boyer, R. y A. Tiberghien (1989): Las finalidades de la enseñanza de la física y la química vistas por profesores y alumnos franceses. Enseñanza de las Ciencias, 7 (3): 213-222.

Bybee, R.W., G.E. Deboer (1994): Research en Goals for the Science Curriculum. Ir Handbook of Research en Science Teaching and Learning (DL. Gabel, ed), Mac-Millan, New York, 357-383.

Bybee, R.W., y N. Ben-zui (1998): Transforming Goals te Practices. In International Handbook of Science Education (B.J.Fraser y KG. Tobin, ed), Kluwer Academic P., Dordrecht, pp 487-498.

Caamaño, A. (AA.VV.) (1995): Monografía: La educación Ciencia-Tecnología-Sociedad. Alambique. Didáctica de las Ciencias Experimentales, 3.

Fleming, R. (1998): Undergraduate science students' views on the relationship between Science, Technology and Society, International Journal of Science Education, 10(4): 449-463.

Furió, C. y A. Vilches (1997): La Enseñanza y el Aprendizaje de las Ciencias de la Naturaleza en la Educación Secundaria. Horsori. Barcelona.

Gil, D., J. Carrascosa, C. Furió y otros (1991): La Enseñanza de las Ciencias en la Educación Secundaria. Horsori. Barcelona.

Hodson, D. (1992): In search of a meaningful relatiorship: an exploration of sorne issues relating te integration ir science and science education. International Journal of Science Education, 14(5): 541-562.

Hodson, D. (1993): Ir search of a Rationale ter Multicultural Science Education. Science Education, 77 (6): 585-711.

López, J. A. (1 998a): Ciencia Tecnelogia y Sociedad. Bibliografía comentada. Revista Iberoamericana de Educación, 18:171-176. 
Martí, J. (1968): Obras Completas: Editorial de Ciencias Sociales, La Habana, t.8.

Penick, J.E. y RE. Yager (1986): Trends ir science education: sorne observations of exemplary programme ir the United States. Eurepear Journal of Science Education, 8 (1): $1-8$.

Ryan, A. 0. (1990): Los efectos de la región, número de asignaturas de ciencias cursadas y sexo sobre la opinión de los estudiantes canadienses en cuestiones de ciencia, técnica y sociedad. Enseñanza de las Ciencias, 8 (1): 3-10.

San Valero, C. y J. Solbes (1995): Bibliografía CTS. Alambique, 3: 69-72.

Schibeci, R. A. (1984): Acttitudes te science: Un update. Studies ir Science Educatien, 11:26-59.

Schibeci, R. A. (1986): magos of science, scientistes and science education. Science Education, 70(2): 139-149.

Simpson, R. Y S. Oliver (1985): Attitude Toward Science and Achievement Motivation Pretiles of Male and Formale Science Students ir Grades Six through Ten. Science Education, 69(4): 511-526.

Simpson, R. Y S. Oliver (1990): A summary of majar irfluences en attitude toward and achievement ir science among adolescent students. Science Education, 74(1): 1-18.

Solbes, J. Y. A. Vilches (1992): El modelo constructivista y las relaciones CTS. Enseñanza de las Ciencias, 10 (2): 181-186.

Solbes, J. Y. A. Vilches (1992): STS interactiors and the teaching of physics and chemistry. Science Education, 81(4): 377-386.

Solbes, J. YA. Vilches (1995): El profesorado y las actividades CTS. Alambique, 3: 30-38.

Salomen, J. (1993): Teaching Science Techrology and Society. Open University Press, Buckingham.

Vázquez, A. y MA. Manasero (1995): Actitudes relacionadas con la ciencia: Una revisión conceptual. Enseñanza de las Ciencias, 13(3): 337-346.

Vázquez, A. y MA. Manasero (1997): Una evaluación de las actitudes relacionadas con la ciencia. Enseñanza de las Ciencias, 15 (2): 199-213.

Vilches, A y Furió, C (1999): Ciencia, Tecnología y Sociedad: sus implicaciones en la educación científica del siglo XXI. PROMET, Editorial Academia, La Habana.

Vilches, A. (1994): Las interacciones ciencia, técnica, sociedad. Selección bibliográfica temática. Enseñanza de las ciencias, 12 (1): 112-120. 